\title{
Microstructural Evaluation in an Instant
}

G.A Blann* G.M. Lucas*

* Buehler, Ltd. Customer Service Laboratory, 41 Waukegan Road, Lake Bluff, IL 60044

In the years prior to the introduction of diamond abrasives and semi-automatic and fully automatic polishing devices, mechanical polishing was a more tedious process that was truly dependent on the skill of the person doing the preparation. Attaining high-quality mechanically polished surfaces routinely was difficult even for the most competent metallographers. The possibility of replacing at least the polishing stages of specimen preparation by a relatively simple electrolytic treatment, which appeared to be capable of producing quality surfaces with good reproducibility, was highly attractive. When microstructural characteristics need to be observed rapidly on a wide range of materials, there is no substitute for electrolytic polishing. It is a tool that is widely used in both the research and production areas of various metal manufacturing facilities. Universally applicable mechanisms have not been developed and acceptable, but continuing studies have produced considerable insight into the phenomena. The literature on electropolishing is vast, with many electropolishing solutions reported for a wide variety of metals.

The type of electrolytes for polishing can be placed in the following eight groups:

- Perchloric acid and alcohol with or without organic additives

- Perchloric \& Acetic acids in varying proportions

- Phosphoric acid in water or organic solvent

- Sulfuric acid in water or organic solvent

- Chromic acid in water

- Mixed acids or salts in water or organic solution

- Alkaline electrolytes

- Ethyl alcohol and nitric acid mixed

If good results are to be duplicated, the metallographer must control many experimental variables. Mixing the electrolytes must be performed with care, since many of the solutions pose a safety hazard if mixed or handled improperly.

Some variables that influence electropolishing results include the following:

- Surface area to be polished

- Specimen orientation

- Choice of cathode

- Ratio of cathode-to-anode surface area

- Composition of specimen including impurities

- Electrolyte bath age and temperature

- Current density and voltage

- Degree of mechanical treatment

Below are of various aluminum sheets that have been electropolished and etched. 




Figure 1. 1100-0 sheet



Figure 3. 1145-H16 sheet

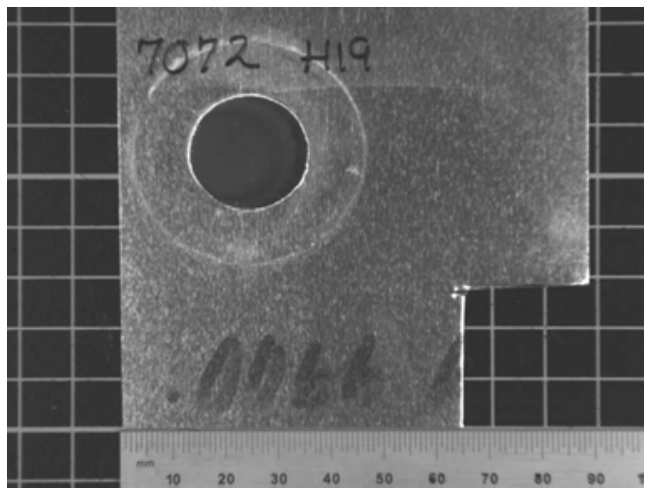

Figure 5. 7072-H19 sheet



Figure 7. 1100-H22 sheet

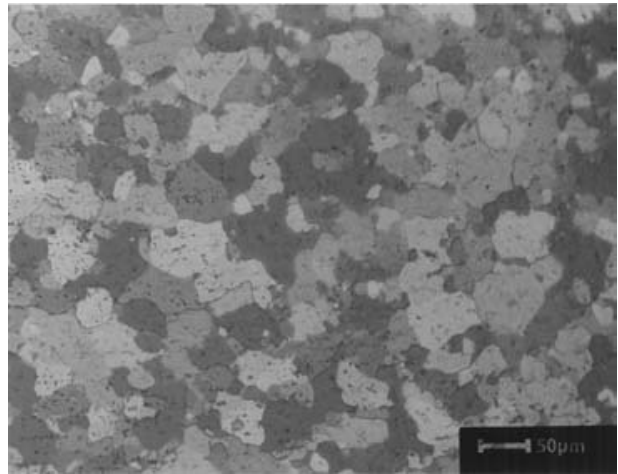

Figure 2. Showing equiaxed grains

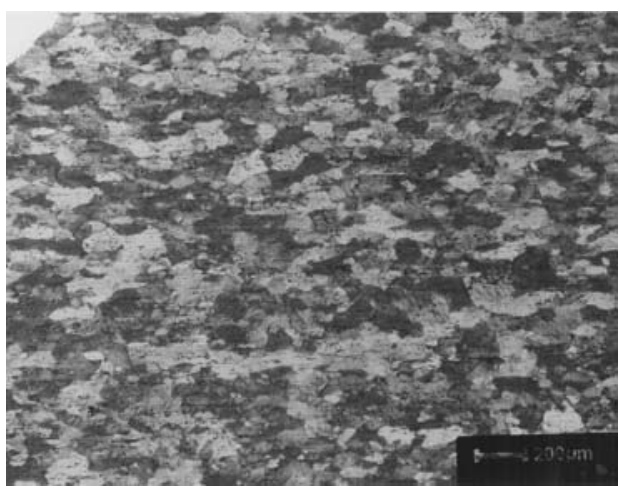

Figure 4. Showing slightly deformed grains



Figure 6. Showing deformed grains

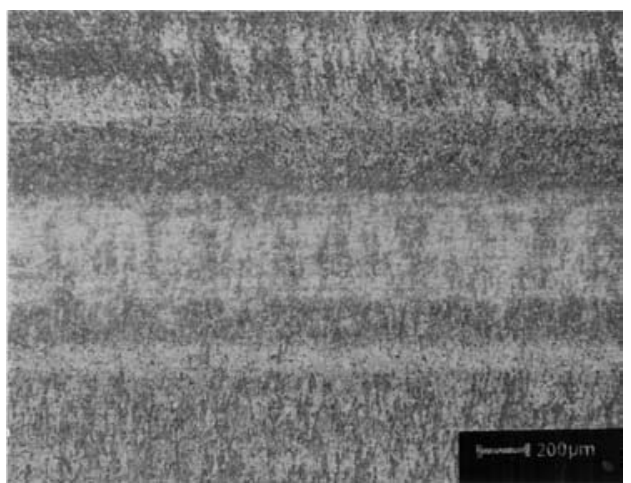

Figure 8 . Showing very deformed grains 